\title{
Miningo
}

\section{Field parametric geostatistics - An alternative to tackle gold grade estimations}

\section{Rochana da Silva Machado ${ }^{1,2}$ \\ http://orcid.org/0000-0001-8904-9211 João Felipe Coimbra Leite Costa ${ }^{1,3}$}

'Universidade Federal do Rio Grande do Sul - UFRS, Departamento de Engenharia de Minas, Porto Alegre - Rio Grande do Sul - Brasil.

E-mails:2rochana.machado@gmail.com ${ }^{3}$ jfelipe@ufrgs.br

\section{Abstract}

High anomalous grades are very common in gold deposits, whose presence requires careful treatment to prevent overestimation of metal content. Mineral resource analysts have worked on the estimation of several gold deposits, and none of the classical methods were able to avoid manual interventions, such as cutting high grades for local estimation or using more information beyond the data for the variogram inference. The Field Parametric Geostatistics (FPG) is presented as an alternative for the application of linear kriging methods to estimate highly skewed distributions, proposing a mathematical model which incorporates the grades and its representativeness into a new variable, reducing the influence of high grades without empirical manual interventions. In this article, the mathematical formulation of the FPG theory is presented, as well as its application in datasets with outliers and high skewed distributions: the Walker Lake dataset and the Amapari gold deposit. The results are compared to results obtained by the application of standard techniques, demonstrating that FPG is a feasible alternative to estimate local grades and local reserves for highly skewed variables.

keywords: kriging, outliers, skewed distribution, gold.

\section{Introduction}

High anomalous grades are very common in mineral deposits of precious metals, such as gold and platinum. The definition of outliers according to Barnett and Lewis (1984) is a datum which seems to be inconsistent with the remaining of the data set.

The presence of outlier grades, very common in gold mineralization, introduces significant variability on statistical parameters and experimental semivariogram calculation. A low number of outliers could be responsible for a metal content overestimation; in other words, a minimum alteration in the number of high grade samples can cause a huge difference between the estimated and mined reserves. Due to their effect on resource estimation, these values should be identi- fied and carefully treated.

There is not a specific procedure universally accepted to deal with outliers. Mineral resource analysts have worked at several gold deposits and in all of them none of the mathematical methods were able to avoid manual interventions, such as cutting high grades for local estimation and using information beyond the data to infer the variograms (Machado et al., 2011; Machado, 2012).

The technique of capping applied to outliers as an attempt to reduce their importance during grade estimation is widespread over the mining industry, based on previous experiences or statistical parameters related to the cumulative distribution function. These methods are subjective and sometime not common sense. Indicator Kriging (IK) (Journel, 1982) was initially developed to estimate probability distributions, but indicator transformation is also an effective way of limiting the effect of very high values (Glacken and Blackney, 1998); however, it is difficult to define spatial continuity to indicators above the $95^{\text {th }}$ percentile of data, due to few samples and sparse spatial distribution. Costa (1997) presents robust kriging in a mathematical framework to map and correct high values that are distinct from the remaining data.

Armony $(2000 ; 2001 ; 2005)$ developed a theory based on mathematical methods to limit the influence of outliers, which explains and justifies the practice of resource analysts of lowering high grades: the Field Parametric Geostatistics (FPG).

\section{The theory of field parametric geostatistics}

Desirable properties that could be inferred from datasets are related to continuity and representativeness. The continuity and representativeness depends on grade variability and the portion represented by each sample. In kriging, continuity is obtained through the modeling of the experimental semivariogram, and it commonly gives the same representativeness to each sample, regardless of analyzed grades.

A theory was developed, based on a variable transformation known as Field
Parametric Geostatistics (FPG). The new variable assigns an area or volume of influence to each sample according to its grade - called extension $(\tau)$ - which incorporates the representativeness during estimation using all the available 
information and reduces the influence of anomalous grades without subjectivity of manual interventions. Machado (2012) and Machado et. al (2012) presented details about the theory and its mathematical formulation.

It is recommended to apply the vari-

\section{Case studies}

The FPG technique is demonstrated by its application on datasets with high-skewed distributions. The first is the Walker Lake dataset (Isaaks able transformation only to the high grade populations: in an earlier study, Armony (2000) verified that applying the transformation to the whole dataset would lead to underestimation. Detection of the limit between two populations can be done by searching for an inflexion point in the cumulative frequency curve, where the angle of the curve changes drastically. The knowledge of the nature of the data and, in the mineral frame, the knowledge of the genesis of the deposit can be very important and sometimes crucial to efficiently apply the FPG technique.

\subsection{Walker lake dataset: variables $\mathrm{U}$ and $\mathrm{V}$}

Analysis of cumulative frequency curves of $\mathrm{U}$ and $\mathrm{V}$ (Figures 1 and 2) show that there is no significant change in the angle of the curve, which means and Srivastava, 1989), where variables $\mathrm{U}$ and $\mathrm{V}$ are derived from a digital elevation model of the Walker Lake area, located in Nevada, USA. The

\begin{tabular}{c|c|c} 
& Count & Mean \\
\hline$U$ & 275 & 604.08 \\
\hline$U($ declustered $)$ & 275 & 555.00 \\
\hline$V$ & 470 & 435.30 \\
\hline$V($ declustered $)$ & 470 & 408.53 \\
\hline
\end{tabular}
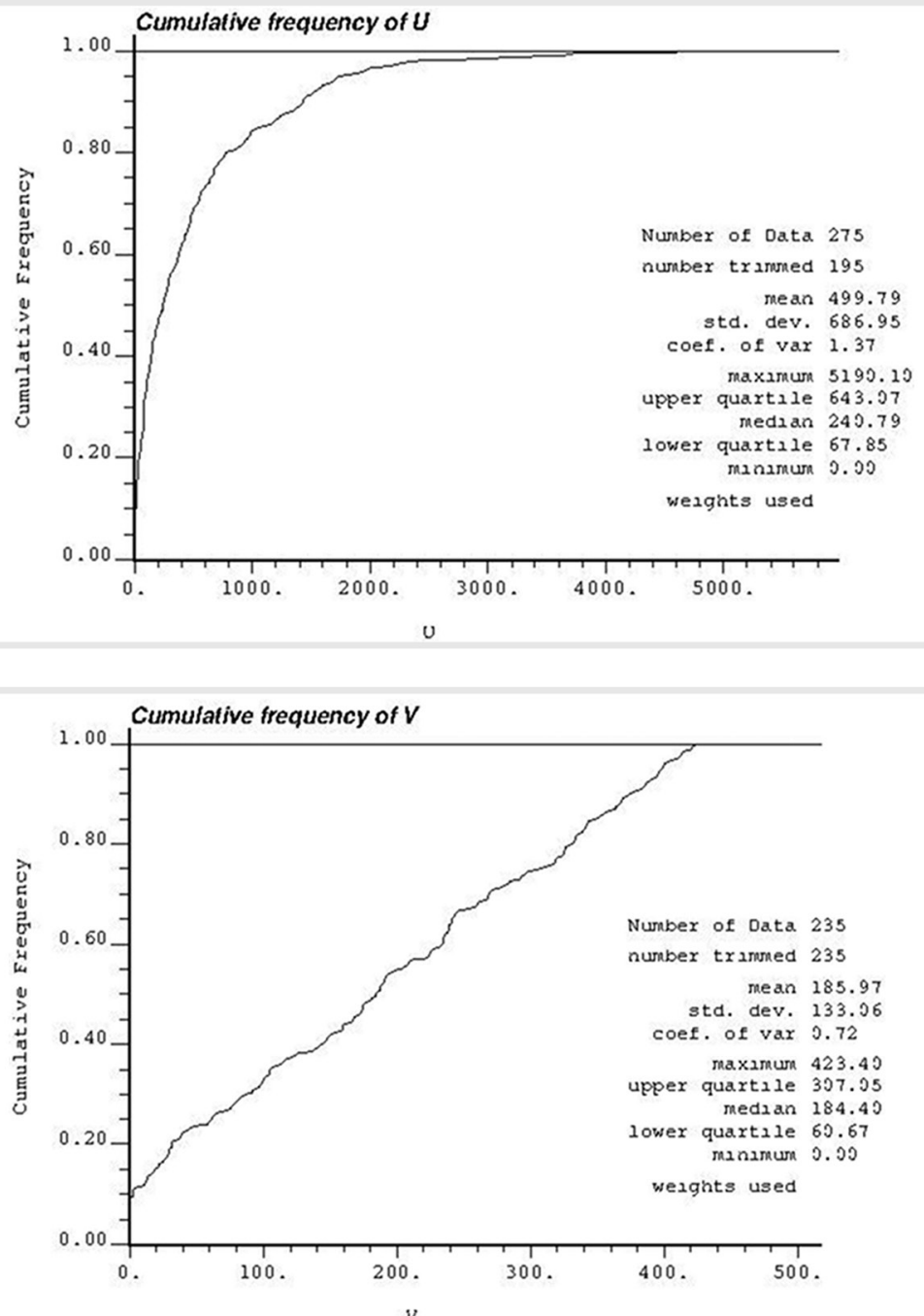

$\mathrm{v}$ second is the Amapari gold deposit, located in Amapá state, Brazil, where the domain of colluvia was chosen to develop this study.

be back transformed to the original population. The statistical parameters for $\mathrm{U}$ and $\mathrm{V}$ variables are presented in Table 1.

Table 1

Statistical parameters for $\mathrm{U}$ and $\mathrm{V}$.

Figure 1

Cumulative frequency distribution of variable $U$.

Figure 2

Cumulative frequency distribution of variable $\mathrm{V}$. 
The grade estimation was performed by Field Parametric Geostatistics (FPG) and its results are compared to other traditional techniques: ordinary kriging of the original values $(\mathrm{OK})$, ordinary kriging after capping the extreme values (capping

Table 2

Statistical parameters for the $U$ and $V$ estimates.

Correlation coefficients for $\mathrm{U}$ and $\mathrm{V}$.

Table 3

The results show that the global average of deposit was best approximated by FPG, especially for the U variable where other applications of kriging lead to overestimation. The difference on the average grade between FPG and the reference model is approximately $4 \%$, while the difference produced on other estimations is up to $96 \%$.

The influence of each method on

Figure 3

Grade vs tonnage curves for $U$ estimates. at p97 and at p90), and median indicator kriging with hyperbolic upper tail extrapolations (E-type 1.5 and E-type 3.0) whose results were post processed with postik from GSLIB (Deutsch and Journel, 1998). The results were also compared against reference values derived from 78000 known as the exhaustive dataset (Reference). The statistical parameters for $U$ and $\mathrm{V}$ estimates are presented in Table 2 and the correlation coefficients are presented in Table 3.

\begin{tabular}{|c|c|c|c|c|c|}
\hline & Count & Mean & & Count & Mean \\
\hline U (Reference) & 3120 & 266.04 & V(Reference) & 3120 & 277.98 \\
\hline U (FPG) & 3120 & 255.22 & $\mathrm{~V}(\mathrm{FPG})$ & 3120 & 261.84 \\
\hline $\mathrm{U}(\mathrm{OK})$ & 3120 & 522.33 & $\mathrm{~V}(\mathrm{OK})$ & 3120 & 292.17 \\
\hline U (capping p97) & 3120 & 477.00 & V (capping p90) & 3120 & 289.43 \\
\hline U (E-type 1.5) & 3120 & 244.86 & V(E-type 1.5) & 3120 & 323.17 \\
\hline U (E-type 3.0) & 3120 & 221.25 & $\mathrm{~V}($ E-type 3.0) & 3120 & 317.80 \\
\hline
\end{tabular}

\begin{tabular}{c|c|c|c|c|c} 
& $\mathrm{U}(\mathrm{FPG})$ & $\mathrm{U}(\mathrm{OK})$ & $\mathrm{U}$ (capping p97) & $\mathrm{U}$ (E-type 1.5) & $\mathrm{U}($ E-type 3.0) \\
\hline $\mathrm{U}$ (Reference) & 0.51 & 0.42 & 0.47 & 0.63 & 0.64 \\
\hline & $\mathrm{V}(\mathrm{FPG})$ & $\mathrm{V}(\mathrm{OK})$ & $\mathrm{V}($ capping p90) & $\mathrm{V}($ E-type 1.5) & $\mathrm{V}($ E-type 3.0) \\
\hline $\mathrm{V}$ (Reference) & 0.87 & 0.85 & 0.83 & 0.83 & 0.83 \\
\hline
\end{tabular}

the resources are also compared using grade versus tonnage curves for $\mathrm{U}$ and $\mathrm{V}$ estimates. In Figure 3 the ordinary kriging of the original values and capping of the extreme values $(\mathrm{U}(\mathrm{OK})$ and $\mathrm{U}($ capping p97)) provide similar curves, but these results depart from the reference model. Estimates using median indicator kriging (U(E-type 1,5) and U(E-type 3,0)) and using FPG technique provided similar curves and closer to the reference model; however the FPG estimate is more conservative after a cutoff grade of 200, compared to the reference model. In Figure 4, although the FPG estimates (V(FPG)) are more adherent to the reference model considering the range from 0 to 400 cutoff grades, the curves provided by other methods do not present large discrepancies in relation to the reference model.

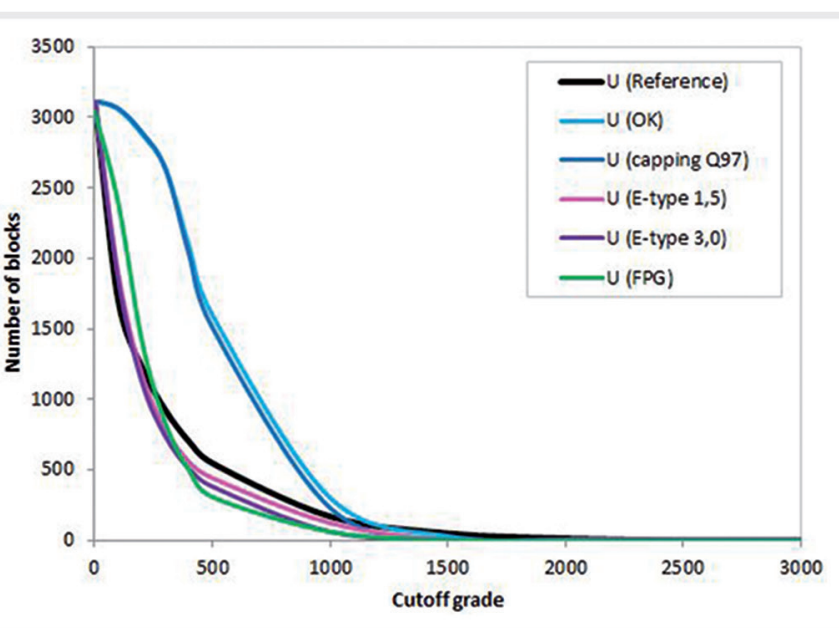




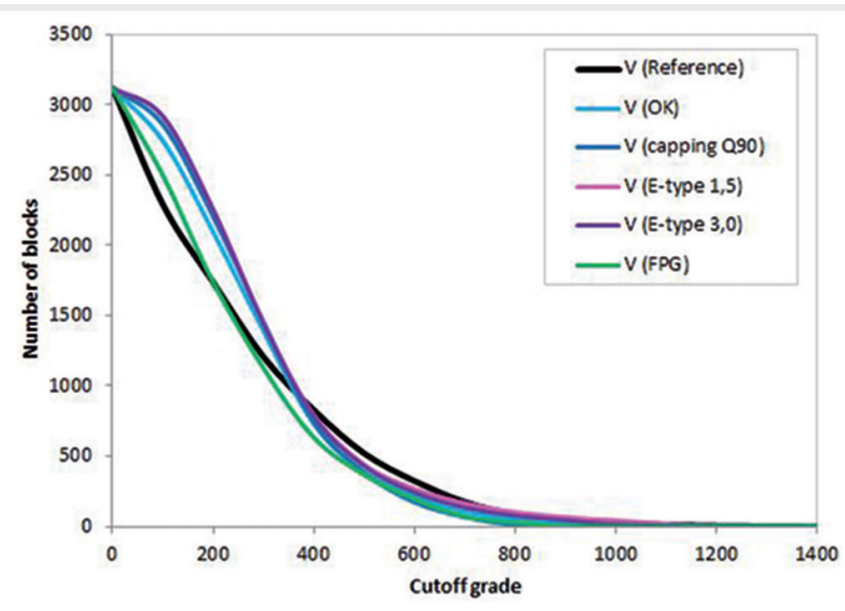

\subsection{Amapari Gold Deposit}

Analyzing the cumulative frequency curve for gold grades from Amapari dataset shows that there are evidences of two populations, which could be separated by the inflexion point around 5 ppm (Figures 5). The population of low grades was estimated using ordinary kriging of the original values; the population of high grades had its values transformed by the FPG technique before estimation by ordinary kriging, and the results were back transformed to the original grade distribution. The probability

Figure 4

Grades vs tonnage curves for $\mathrm{V}$ estimates.

of each block to belong to a high grade or low grade population was estimated using indicator kriging, and these proportions were used to combine the estimation of each population to assign the final grade to the blocks.

Figure 5

Shape of the cumulative frequency of gold grades in the Amapari deposit (two populations).

A division of colluvia domain into northern and southern portions was (a)

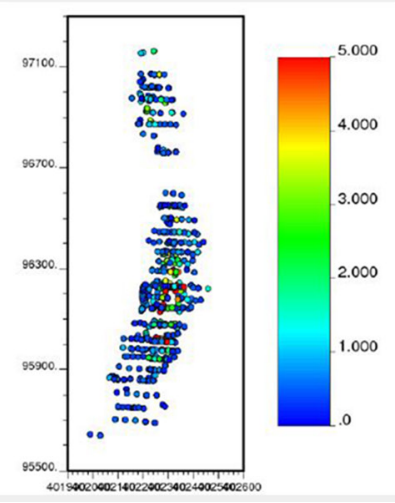

performed (Figure 6), whose statistics are presented in Table 4. (b)

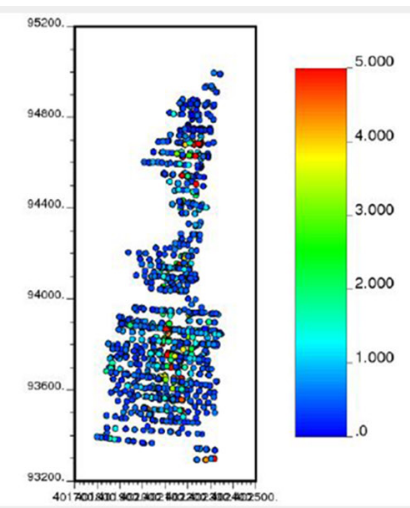

\begin{tabular}{c|c|c|c|c|c|c} 
& Region & Count & Mean & Region & Count & Mean \\
\hline Au, ppm & North & 1730 & 1.47 & South & 4127 & 1.50 \\
\hline Au (declustered), ppm & North & 1730 & 0.90 & South & 4127 & 0.97 \\
\hline
\end{tabular}

Figure 6

Location map of Au samples from Amapari gold deposit: (a) northern portion and (b) southern portion of the deposit. The color scale is presented from 0 ppm to 5 ppm due to the highly skewed population.

Table 4

Descriptive statistics for Au. 
At the Amapari gold deposit, the grade estimation was also performed by Field Parametric Geostatistics (FPG) and its results were compared to ordinary kriging of the original values $(\mathrm{OK})$, or-

Table 5

Descriptive statistics for Au estimates.

The average grade of FPG estimates is more conservative than the average estimated by other methods, both in the northern and southern portion of the colluvia deposit. This is assigned to a more effective control of outliers on the FPG estimates.

Figure 7

Grade vs tonnage curves for Au estimates in the northern portion.

Figure 8

Grade vs tonnage curves for Au estimates in the southern portion.

\section{Conclusions}

Field Parametric Geostatistics can be considered a robust alternative for grade estimation, reducing the impact of outliers without the subjectiviness dinary kriging after capping the extreme values from datasets (capping p95 and capping p98) above the 95th and the 98th percentile of the distribution $(\mathrm{Au}=5.03$ ppm and $\mathrm{Au}=9.20 \mathrm{ppm}$, respectively), and median indicator kriging with hyperbolic upper tail extrapolations (E-type 1.5 and E-type 4.0). The statistical parameters for Au estimates are presented in Table 5 .

\begin{tabular}{c|c|c|c|c|c|c} 
& Region & Count & Mean & Region & Count & Mean \\
\hline Au (FPG), ppm & North & 155488 & 0.59 & South & 226619 & 0.62 \\
\hline Au (OK), ppm & North & 155488 & 0.68 & South & 226619 & 0.88 \\
\hline Au (capping p98), ppm & North & 155488 & 0.65 & South & 226619 & 0.77 \\
\hline Au (capping p95), ppm & North & 155488 & 0.63 & South & 226619 & 0.71 \\
\hline Au (E-type 1.5) & North & 155488 & 0.75 & South & 226619 & 0.86 \\
\hline Au (E-type 4.0) & North & 155488 & 0.66 & South & 226619 & 0.70 \\
\hline
\end{tabular}

Grade versus tonnage curves for $\mathrm{Au}$ estimates were generated to compare the estimated models to the curve obtained by samples after a change of support (affine correction). Figures 7 and 8 show that the use of capping or median indicator kriging to control the effect of extreme values was not effective, given that their curves are coincident to ordinary kriging of raw data. The application of FPG provided better results, reducing the influence of outliers during estimation.
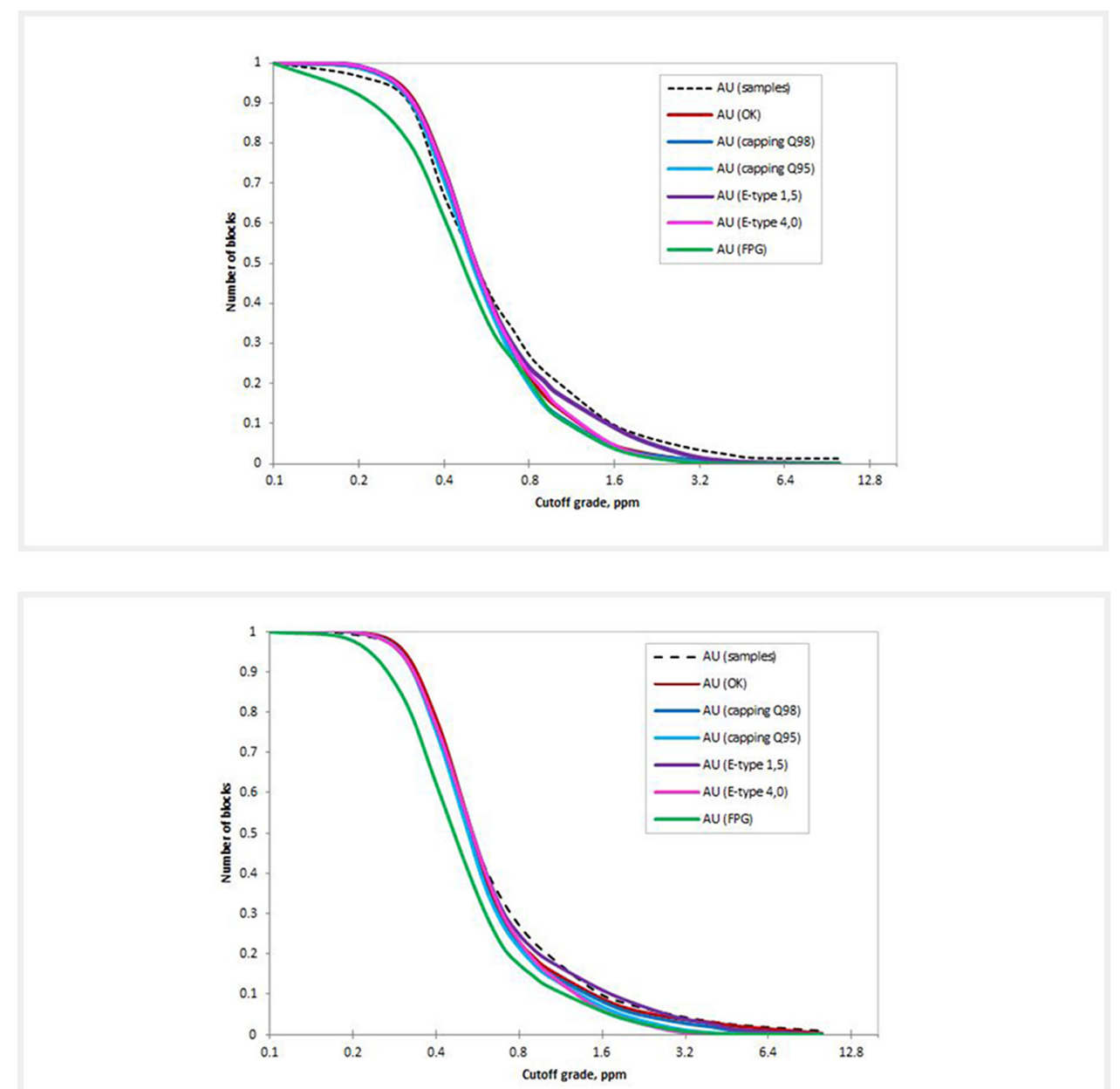

of choosing restriction parameters. The effects were demonstrated through good results obtained on $\mathrm{U}$ estimates in comparison to the reference model, and the effective control of high grades of $\mathrm{Au}$ compared to the classical approach used in the mining industry. 


\section{References}

ARMONY, M. Geoestatística paramétrica de campo. Rio de Janeiro: UFRJ, 2000.

ARMONY, M. Field parametric geostatistics. International Journal of Surface Mining, n. 15, p. 100-122, 2001.

ARMONY, M. Logical basis for field parametric geostatistics. International Journal of Surface Mining, n. 19, p. 144-157, 2005.

BARNETT, V. \& LEWIS, T. Outliers in Statistical Data. 2nd ed. Chichester: John Wiley $\&$ Sons Ltd, 1984, 365p.

COSTA, J.F.C.L. Developments in recoverable reserves and ore body modeling. Queensland: The University of Queensland, 1997.

DEUTSCH, C. V. \& JOURNEL, A. G. GSLIB: Geostatistical Software Library and User's Guide. New York: Oxford University Press, 1998, 369 p.

GLACKEN, I. M. \& BLACKNEY, P. A. A practitioners implementation of indicator kriging In: Beyond Ordinary Kriging - Non-Linear Geostatistical Methods in Practice, 1998. Proceedings of ..., Perth: Geostatistical Association of Australasia, 1998, p. 26-39.

ISAAKS, E. H. \& SRIVASTAVA, R.M. An introduction to applied Geostatistics. New York: Oxford University Press, 1998, $547 \mathrm{p}$.

JOURNEL, A. G. The indicator approach to estimation of spatial distributions. In: APCOM, 17, 1982. Golden. Proceedings of ..., Golden: Curran Associates, 1982, p. 793-806.

MACHADO, R. S., ARMONY, M., COSTA, J. F. C. L., KOPPE, J. C. FPG - Geostatistical rigorous solutions for highly-skewed distributions. In: GEOMIN, 2, 2011. Antofagasta. Proceedings of..., Santiago: Gecamin Ltd., 2011, p. 1-9.

MACHADO, R. S., ARMONY, M. and COSTA, J. F. C. L. Field Parametric Geostatistics - a rigorous theory to solve problems of highly skewed distributions. In: GEOSTATS, 9, 2012. Oslo. Proceedings of..., Oslo: Springer, 2012, p. 383-396.

MACHADO, R. S. Uma alternativa para a estimativa de teores em depósitos de ouro: Geoestatística Paramétrica de Campo. Porto Alegre: UFRGS, 2012.

Received: 27 May 2018 - Accepted: 27 September 2018. 